1407

\section{INCIDENCE AND RISK FACTORS FOR HEALTHCARE-ASSOCIATED PNEUMONIA IN A PEDIATRIC INTENSIVE CARE UNIT}

\author{
R.J.A. Casado1, M.J.G. Mello ${ }^{1,2}$, R.C.F. Aragão, ${ }^{1,3}$,
} M.D.F.P.M. Albuquerque ${ }^{4,5}$, J.B. Correia ${ }^{1,3}$

1 Instituto de Medicina Integral Professor Fernando Figueira, ${ }^{2}$ Faculdade Pernambucana de Saude, ${ }^{3}$ Universidade de Pernambuco, ${ }^{4}$ Centro de Pesquisas Aggeu Magalhaes - FIOCRUZ, ${ }^{5}$ Universidade Federal de Pernambuco, Recife, Brazil

Backgorund and aims: Healthcare-associated pneumonia (HCAP) is one of the top two causes of healthcare associated infections ( $\mathrm{HAl})$ in pediatric intensive care units (PICU). This study aimed to determine the incidence and risk factors for HCAP in a PICU.

Methods: prospective cohort study in a paediatric intensive care unit with 16 medical and surgical beds in a tertiary teaching hospital in Recife, northeast Brazil. Patients aged less than 18 years were consecutively enrolled between January 2005 and June 2006. Newborns and patients admitted for surveillance and those staying for less than 24 hours were excluded. Patients were followed up daily throughout the stay and until $48 \mathrm{~h}$ after discharge from the unit.

Results: There were 765 eligible admissions. HCAP occurred in 51 (6.7\%) patients, with an incidence density of 13.1 episodes/1000 patient-days. There were $366(47.8 \%)$ patients on mechanical ventilation, of whom $39(10.7 \%)$ presented with ventilator-associated pneumonia, with an incidence density of 27.1/1000 days on ventilation. Longer stay on ventilation $(\mathrm{OR}=1.04 \mathrm{IC} 95=1.01-1.08)$, use of gastric tube $(O R=2.88$ IC95=1.41-5.87) and of sedatives/analgesics $(\mathrm{OR}=2.45 \quad \mathrm{IC} 95=1.27-4.72)$ were identified as independent risk factors for healthcare-associated pneumonia.

Conclusions: identification of independent predictors of healthcare-associated pneumonia may inform preventive measures. Strategies to optimize use of sedatives/analgesics, reduce the use of gastric tubes and the time on ventilation should be considered for inclusion in future intervention studies.
1408

\section{EPIDEMIOLOGY OF EARLY-ONSET SEPSIS IN A NICU DURING A 12-YEAR PERIOD (1996- 2007)}

E. Diamanti, K. Sarafidis, V. Soubasi, K. Agoropoulou, K. Anegronou, E. Agakidou, A. Marioglou, V. Drossou-Agakidou

1st Dept. of Neonatology, Aristotle University of Thessaloniki, Thessaloniki, Greece

Aim: To determine the incidence and the causative pathogens of early-onset sepsis (EOS $\leq 3^{\text {rd }}$ day of life).

Patients-methods: We prospectively studied the perinatal and neonatal characteristics of all neonates who were born in our perinatal center and were treated in our department, as well as all neonates admitted in our tertiary NICU from the area of Macedonia and Thessaly, during the period 1996-2007. Neonates with positive blood culture were further studied according to the time of the $1^{\text {st }}$ sepsis episode and causative pathogen isolated.

Results: During the study period, 9113 neonates were admitted to the NICU. Of them, EOS had 229 $(2.5 \%)$ neonates. In the $73.4 \%$ of them, gram positive bacteria were isolated. Most common causative pathogens were: Coagulase negative Staphylococci (48.5\%), Escherichia coli (11.8\%), Staphylococcus aureus $(7.9 \%)$, Klebsiella $(5.2 \%)$, GBS $(4.8 \%)$, Streptococci (other, 4.8\%), Enterococcus (3.9\%), Pseudomonas (3.1\%), Enterobacteria (2.2\%), Acinetobacter (2.2\%), Streptococcus viridans (1.3\%), Candida albicans (1.3\%), Listeria (0.9\%), Serratia (0.4\%), Hemophilus (0.4\%), Streptococcus pneumonia $(0.4 \%)$ and Brucella melitensis (0.4\%). Of the 20.643 neonates born alive at our center, $101(0.49 \%)$ had EOS. Also, EOS developed in $3.1 \%$ of the inborn neonates with $\mathrm{BW}<1500 \mathrm{~g}$, and $2.7 \%$ with $\mathrm{GA}<33 \mathrm{wks}$, of whom $45.9 \%$ and $51.4 \%$, respectively, had EOS from gram negative and positive bacteria.

Conclusion: The most common bacteria for EOS in our NICU were staphylococci sp., whereas GBS and Listeria are not important in the epidemiology of EOS. Gram negative bacteria are involved mostly in inborn babies with $\mathrm{GA}<33$ wks. 